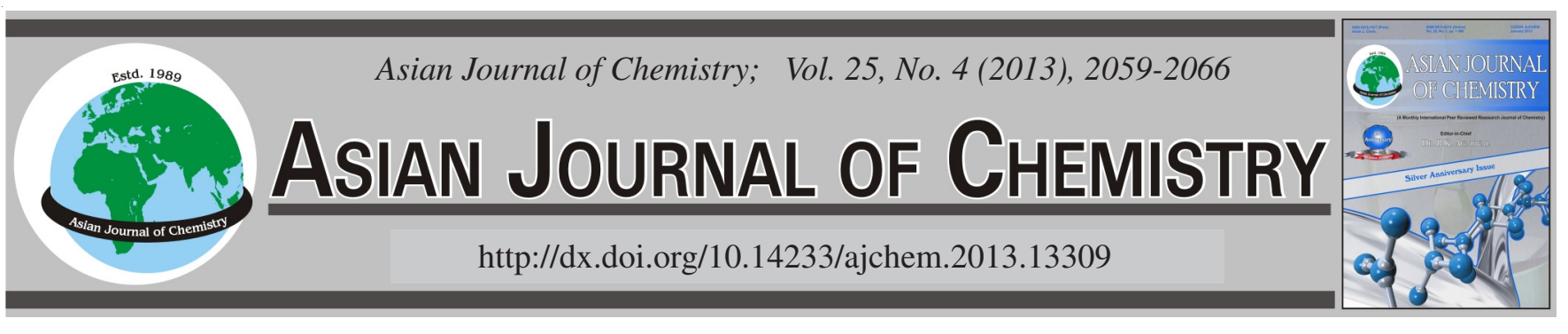

\title{
Multivariate Statistics and Heavy Metals Contamination in Beach Sediments from The Sakarya Canyon, Turkey
}

\section{Gurhan Yalcin ${ }^{1, *}$, Gunseli Simsek $^{2}$, Sema Bilge OcaK ${ }^{3}$, Fusun Yalcin $^{4}$, Yakup Kalayci ${ }^{5}$ and M. Erkan Karaman ${ }^{1}$}

\author{
${ }^{1}$ Department of Geological Engineering, Faculty of Engineering, Akdeniz University, 07058 Antalya, Turkey \\ ${ }^{2}$ Department of Geological Engineering, Faculty of Engineering, Nigde University, 51200 Nigde, Turkey \\ ${ }^{3}$ Gazi University, Atatürk Junior Technical College, Ankara, Turkey \\ ${ }^{4}$ Department of Mathematics, Faculty of Science, Akdeniz University, 07058 Antalya, Turkey \\ ${ }^{5}$ Ankara Nuclear Research and Training Center, 06100 Besevler, Ankara, Turkey
}

*Corresponding author: E-mail: gurhanyalcin@akdeniz.edu.tr; gurhan46@gmail.com

(Received: 22 December 2011;

Accepted: 12 October 2012)

AJC-12279

\begin{abstract}
The aim of the study is to determine heavy metal contents and their possible origins that represent the variability of The Sakarya Canyon coastal sediments. In addition to determine the source of heavy metals (natural and anthropogenic), simple and multivariate statistical analyses were applied to the samples. In all the samples, ignition loss ratio is between $0.01-0.09 .47 .26 \%$ of the samples, which have 0.5 $0.25 \mathrm{~mm}$, show very good sorting. G10, G19, G20 and G21 reflect the conditions of the irregular sedimentary environment. The heavy metals, $\mathrm{Fe}, \mathrm{Mg}, \mathrm{Ti}, \mathrm{Cr}, \mathrm{Zn}, \mathrm{Pb}$ and $\mathrm{Cu}$, are considered to come from near regions according to frequency histograms. By principal component analysis (PCA; factor 1: $40.911 \%$; factor 2: $21.558 \%$; factor 3: $13.548 \%$ ) and cluster analysis, heavy metals were formed three (3) groups. According to hierarchical cluster analysis, Q-type cluster at the similarity level of $50 \%$ form three (3) different groups and they show the same features during pollution. These results reveal that they are highly reliable data for statistical data of model summary (according to the value $\mathrm{R}^{2}=100$ ) and Anova 21 explanation value. According to maximum abundances As:G4; Ni:G7; Mg, Ti, $\mathrm{Mn}, \mathrm{Fe}, \mathrm{V}, \mathrm{Cr}, \mathrm{Co}, \mathrm{Nb}: \mathrm{G} 13 ; \mathrm{Cu}, \mathrm{Zr}, \mathrm{Sn}: \mathrm{G} 20 ; \mathrm{Al}, \mathrm{Zn}, \mathrm{Ga}, \mathrm{Cd}, \mathrm{Pb}: \mathrm{G} 22$ stations showed the highest anomaly. Influence of anthropogenic can be constituted in this region coming from port wastes, mining operations, road pollution, urban wastes and industrial wastes.
\end{abstract}

Key Words: Heavy metal, Multivariate statistic, Beach sand, Multivariate, Sakarya Canyon.

\section{INTRODUCTION}

Heavy metal aggregations at sand provide important information in terms of accumulation erosion movements, coastal dynamics and processes ${ }^{1-3}$. Heavy metals have a great ecological significance due to their toxicity and accumulation ${ }^{4}$. In sand dunes, natural and anthropogenic contamination must be determined accurately. Geochemistry and mineralogy of the samples show the effect of both natural and anthropogenic inputs to the catchment. Anthropogenic inputs of natural processes are more dominant than concentrating metals ${ }^{4}$. Geological/ anthropogenic sources of heavy metals in explanation are the most widely used method in the form of multivariate statistical methods ${ }^{5}$. There are several scientific studies in the Black Sea region, e.g., trace elements in macroalgaes of the Black Sea coast, algae and sediments of the Black Sea coast ${ }^{2,3}$ and the Yenice and Sakarya rivers ${ }^{6}$; biological impression of heavy metals at the Western Black Sea ${ }^{7}$; the heavy metal contents of sediments at the Eastern Black Sea in Trabzon region ${ }^{8}$; the heavy metal contents of samples at the Black Sea naval base"; heavy metals contents of sediments in our scope of research field $^{10}$; the Black geochemistry of heavy metals of drainage areas in the Black Sea region ${ }^{11}$; geochemistry and sediment of sea floor sediments to sedimentology in the Central Black Sea region ${ }^{12}$; anthropogenic impact of the Sakarya River ${ }^{13}$ and geology of western Black Sea and Marmara region ${ }^{14}$.

Anthropogenic effects of the rivers on the Black Sea have been studied in the research field. The effects of sulphuric oxide suboxide and anoxic sulfidic collapsing the waters of the Black Sea naval base have been studied ${ }^{15}$. However, the Sakarya Conyon which has the heavy metal contents, its origin and its distribution have not been studied.

In this study, grain size distribution of beach sands in the Sakarya Canyon, heavy metal contents and their geological/ anthropogenic origins and distributions were determined. Multivariate statistical methods were applied to the results of chemical analysis of heavy metals.

\section{EXPERIMENTAL}

The study area is between Cebeci (Kocaeli) and Eregli (Zonguldak) which are located in the north of Turkey and representing the whole Sakarya Canyon. The Canyon represents 
about $110 \mathrm{~km}$ coastline where the human activity is present. The Guluc Stream in Eregli region is the boundary in the study area (Fig. 1). The north of the region is covered with mountain ranges. The region extending from the south to the north constitutes Kocaeli peneplane. Sakarya mountains consist of Keremali Mountain (1543 m), Karadag (1467 m) and Camdagi (880 $\mathrm{m})$. The ratio of the mountains is $34 \%$, plateaus $44 \%$ and 22 $\%$ plain in the region. The Sakarya river, the Maden Creek, Melen stream, Akcakoca stream, Alapli Creek, Guluc stream are located in this region. There are manganese (Geyve district), lead-copper-zinc (Hendek town), iron mines (Karasu town) (30.5 million tons of silica, 78.7 thousand tons of carbonate) in Sakarya region. There are 200.000 tons of manganese mine in Duzce region. Sakarya region has the climate characteristics of the Marmara and the Black Sea. This causes the moist and temperate climate. Winters are rainy and less cold, while summers are hot. The average annual precipitation is 1025.8 $\mathrm{mm}$. The average number of snow-covered day is 5 , the maximum snow depth is $15 \mathrm{~cm}^{16}$.

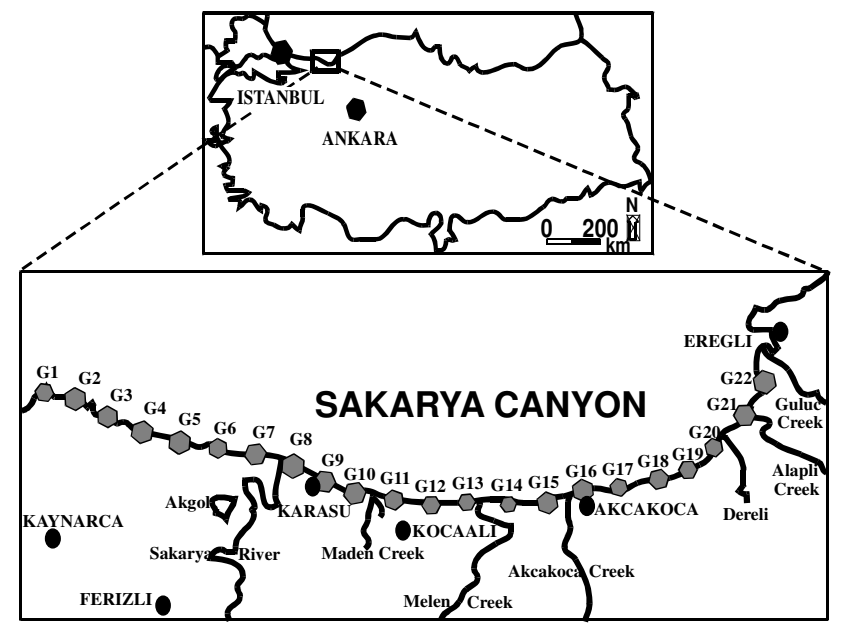

Fig. 1. Areas of the present study (Sakarya Canyon)

Istanbul terane is a continental fragment, $400 \mathrm{~km}$ long and $55 \mathrm{~km}$ wide on the south-western margin of the Black sea. It has a late Precambrian crystalline basement characterized by gneiss, amphibolite, metavolcanic rocks, metaophiolite and voluminous Late Precambrian granitoids $s^{17,18}$. There are marked stratigraphic differences between the western and eastern parts of the Istanbul terane. The Istanbul terrane is separated from the Sakarya terane by the Intra-Pontide suture marking the trace of the Intra-Pontide ocean ${ }^{19}$. During the Carboniferous the Intra-Pontide ocean probably formed the eastern extension of the Rheic ocean ${ }^{20}$ and closed to the collision of the Istanbul and Sakarya teranes in the mid carboniferous. The different Mesozoic stratigraphies of the Istanbul and Sakarya terranes suggest that the Intra-Pontide ocean reopened during the Triassic only to close again in the mid-Cretaceous ${ }^{21}$. The Sakarya zone is situated in the south of the Istanbul-Zonguldak Unit. The basement of the Sakarya zone consists of two different metamorphic associations ${ }^{14}$ : the Uludag and the Yenisehir groups $^{22}$. The Uludag Group is composed of high-grade schists, gneisses, amphibolites and migmatites that are intruded by a granitic. These are unconformably overlain by Permian limestones, Triassic carbonates and clastic rocks "Karakaya formation" $^{23}$ that are also imbricated with ophiolite rocks ${ }^{24}$. The Yenisehir group is composed of a meta ophiolite and a metamorphosed volcanic-sedimentary unit. The two metamorphic associations are collectively overlain by a thick Liassic-Ypresian sedimentary sequence "Bayirkoy, Bilecik, Sogukcam, Vezirhan formations and Golpazari Group"22. Beginning in Middle Eocene time, shallow marine carbonate and clastics were deposited as products of a new regional transgression on the Sakarya zone ${ }^{14}$.

Sand samples were collected from the Sakarya Canyon in October 2010. Each sample was photographed and was determined with Garmin brand GPS-12CX device and marked on 1/100.000 scaled map. Samples from a total of 22 locations were collected at a depth of $0-10 \mathrm{~cm}$ from the surface with the use of the hard plastic shovel and stored in $2-5 \mathrm{~kg}$ plastic bags. Samples were heated at $105^{\circ} \mathrm{C}$ for $24 \mathrm{~h}$. Following the drying of sediment samples, they were homogenized in an agate mortar and pulverized to $2 \mathrm{~mm}>$ grain size. The mortar was washed with $6 \mathrm{~mol} \mathrm{~L}^{-1} \mathrm{HNO}_{3}$ and rinsed with distilled water and dried before each sample process. All samples were transformed to double sided film tablets in $32 \mathrm{~mm}$ diameter and their heavy metal and major oxide contents were analyzed with Spectro Xepos Bechtop X-Ray fluorescence spectrometer. Results are given as \% and $\mathrm{mg} / \mathrm{kg}$. Grain size distribution of beach sand samples was obtained from $100 \mathrm{~g}$ to measure. These samples were received, $105^{\circ} \mathrm{C}$, dried in the oven for 24 h. Then the samples $(\mathrm{mm})(>4),(4.0-2.0)(2.0-1.0)(1.0-0.5)$ (0.5-0.25), (0.25-0.125), (0.125-0.0625), $(0.0625<)$ sieves were numbered. Selection process was carried out for $20 \mathrm{~min}$ with shaking sieve. Every last one of a sieve and the amount of weight is measured by sensitive balance. The measured values are converted into graphics with the Excel software.

Hundred grams of sample from whole samples was chosen and dried for 10 days in furnace under normal circumstances. This process was carried out at $105^{\circ} \mathrm{C}$ for $24 \mathrm{~h}$. Then re-measured grain size of samples was calculated loss of ignition. And dried for chemical analysis $(0.125<)$ numbered approximately 10 $\mathrm{g}$ samples of the sieve became homogenized in an agate mortar. The mortar was washed with $6 \mathrm{~mol} \mathrm{~L}^{-1} \mathrm{HNO}_{3}$ and rinsed with distilled water and dried before each sample process. All samples were transformed to double sided film tablets in 32 mm diameter and their heavy metal and major oxide contents were analyzed with Spectro Xepos Bechtop X-Ray fluorescence spectrometer. Chemical analyses were carried out with brand PanAnalytical Advance Axios Wavelength Dispersive X-Ray fluorescence spectrometer (WDXRF) while the analysis of major elements were done, samples were mixed with the flux rate material 1:10 $\left(66 \% \mathrm{Li}_{2} \mathrm{~B}_{4} \mathrm{O}_{7}+34 \% \mathrm{LiBO}_{2}\right)$ and done glass in the induction furnace. Trace elements during analysis, after $3 \mathrm{~g}$ sample of $27 \mathrm{~mm}$ tablet was developed into a die by applying pressure of 30 tons with the team. In interpreting the origins, it is important to determine the relationship between the groups that heavy metals form among themselves or with each other ${ }^{25}$. Since there are partial differences in surface soil through the depth, geochemical interpretation of these is needed ${ }^{5}$. Therefore, multivariate statistical methods and simple statistical parameters were applied the obtained values. These transactions were made with the SPSS software ${ }^{26}$ and the maps were drawn using the Freehand software. 


\section{RESULTS AND DISCUSSION}

Ignition loss values were calculated to determine the percentage of organic matter, water and moisture (Table-1). Samples which ignition loss ratio is between 0.01 and 0.09 , $3.89 \%$ of coarse gravel-pebble, $3.84 \%$ of very coarse sand, $16.95 \%$ of coarse sand, $47.26 \%$ of medium sand, $27.03 \%$ of fine sand, $0.78 \%$ of very fine sand, $0.21 \%$ of silt + clay is

TABLE-1

IGNITION LOSS VALUES (g)

\begin{tabular}{cccc}
\multicolumn{4}{c}{ IGNITIONLOSS VALUES $(\mathrm{g})$} \\
\hline S. No. & $\begin{array}{c}\text { Before ignition } \\
\text { weight }\end{array}$ & $\begin{array}{c}\text { After ignition } \\
\text { weight }\end{array}$ & Ignition loss \\
\hline 1 & 100.00 & 99.004 & 0.996 \\
2 & 100.00 & 99.681 & 0.319 \\
3 & 100.00 & 99.863 & 0.137 \\
4 & 100.00 & 99.886 & 0.114 \\
5 & 100.00 & 99.843 & 0.157 \\
6 & 100.00 & 99.484 & 0.516 \\
7 & 100.00 & 99.887 & 0.113 \\
8 & 100.00 & 99.835 & 0.165 \\
9 & 100.00 & 99.843 & 0.157 \\
10 & 100.00 & 99.988 & 0.012 \\
11 & 100.00 & 99.990 & 0.01 \\
12 & 100.00 & 99.743 & 0.257 \\
13 & 100.00 & 99.965 & 0.035 \\
14 & 100.00 & 99.765 & 0.235 \\
15 & 100.00 & 99.963 & 0.037 \\
16 & 100.00 & 99.888 & 0.112 \\
17 & 100.00 & 99.916 & 0.084 \\
18 & 100.00 & 99.911 & 0.089 \\
19 & 100.00 & 99.572 & 0.428 \\
20 & 100.00 & 99.984 & 0.016 \\
21 & 100.00 & 99.906 & 0.094 \\
22 & 100.00 & 99.675 & \\
\hline
\end{tabular}

concentrated (Table-2). From these examples, the highest values are $\mathrm{G} 1: 0.996$ and $\mathrm{G} 6: 0.516$, the lowest values are $\mathrm{G} 11$ : 0.01 and the G20:0.016. Particle size distribution is presented in Table-2. G3, 5, 6, 8, 9 and 22 of samples are focused on the fifth column. G17 is dense in the sixth column. All of these locations offering a single type of aggregation has shown a very good sorting. Sample G1, 2, 4, 7, 13 and 14 on the fifth and on the sixth column; G11, on the fourth and fifth column; G16, third and on the fourth column are dense and present negative skew offer fine. G12, 15 and 18 of samples are dense in the fourth, fifth and sixth column. The major rivers flowing to the study area can be listed as the Melen Stream, the Sakarya river $\left(16.7 \times 106 \mathrm{~m}^{3} /\right.$ day $)$, the Akcakoca Creek, the Alapli stream. The river inputs near the locations affect the distribution of sediment. Accordingly, the G20 and G21 samples provide Bimodal distribution. G10, shows bad sorting by dispersing into different columns. G19, offers ill-sorted, negative and a large skew. River's system affecting the region influence uneven ambient conditions.

Heavy metal contents of beach sand in the Sakarya Canyon are given in Table-3. The results of chemical analysis are analyzed statistically (Table-4). The highest values of chemical analysis results are Fe, $\mathrm{Al}, \mathrm{Mg}, \mathrm{Ti}, \mathrm{Mn}, \mathrm{Zn}, \mathrm{Cr}, \mathrm{V}, \mathrm{Zr}$, $\mathrm{Ni}, \mathrm{Pb}, \mathrm{Co}, \mathrm{Cu}, \mathrm{Nb}, \mathrm{As}, \mathrm{Ga}, \mathrm{Sn}, \mathrm{Cd}$. Arithmetical averages are 13121.25, 49950.83, 3544.17, 709.96, 27246.25, 71.91, $128.62,11.13,34.60,9.06,143.17,8.85,8.75,98.23,7.74$, $3.06,3.29,13.31$, respectively and presence rates of minimummaximum are, 4560-40720, 34850 -74400, 1570-13840, 3641816, 14600-75880, 27.5-267.8, 19.4-631.6, 4.3-26, 9.1-78.3, 5.1-18.2, 42.9-783.3, 5.8-12.8, 4.8-13.2, 66.2-162.7, 4.5-17.3, 1.4-5.8, 1.4-6.2, 5.1-37.7, respectively (Fig. 2). The samples have a maximum value of the locations of some heavy metals

\begin{tabular}{|c|c|c|c|c|c|c|c|c|c|}
\hline \multirow{3}{*}{ Coast name } & \multicolumn{8}{|c|}{$\begin{array}{c}\text { TABLE-2 } \\
\text { GRAIN SIZE DISTRIBUTIONS PASSING SIEVE }\end{array}$} & \multirow[b]{2}{*}{$\begin{array}{l}\text { Silt }+ \\
\text { clay }\end{array}$} \\
\hline & \multirow{2}{*}{$\begin{array}{l}\text { S. } \\
\text { No. }\end{array}$} & Gravel & $\begin{array}{l}\text { Coarse } \\
\text { gravel- } \\
\text { pebble }\end{array}$ & $\begin{array}{l}\text { Very coarse } \\
\text { sand }\end{array}$ & $\begin{array}{l}\text { Coarse } \\
\text { sand }\end{array}$ & $\begin{array}{l}\text { Medium } \\
\text { sand }\end{array}$ & Fine sand & Very fine sand & \\
\hline & & $>$ & $(4.0-2.0)$ & $(2.0-1.0)$ & $\begin{array}{l}(1.0- \\
0.5)\end{array}$ & $\begin{array}{l}(0.5- \\
0.25)\end{array}$ & $\begin{array}{l}0.25- \\
0.125 \\
\end{array}$ & $\begin{array}{l}(0.125- \\
0.0625)\end{array}$ & $(<)$ \\
\hline Uzunkum coast & G1 & 0 & 0 & 0.17 & 1.80 & 32.07 & 65.25 & 0.02 & 0.12 \\
\hline Babali coast & G2 & 0 & 0 & 0 & 1.36 & 61.69 & 36.80 & 0.15 & 0 \\
\hline Camitepe village coast & G3 & 0 & 0 & 0.01 & 17.26 & 78.35 & 4.38 & 0 & 0 \\
\hline Denizkoy village coast & G4 & 0 & 0.03 & 0.21 & 5.94 & 58.86 & 34.71 & 0.13 & 0.01 \\
\hline Denizkoy village coast & G5 & 0 & 0.03 & 0.68 & 12.35 & 73.14 & 13.57 & 0.02 & 0.01 \\
\hline Denizkoy village coast & G6 & 0 & 0 & 0.24 & 18.47 & 70.74 & 10.52 & 0.01 & 0 \\
\hline Denizkoy village coast & G7 & 0 & 0.37 & 0.18 & 2.35 & 53.69 & 41.70 & 1.69 & 0.01 \\
\hline Karasu port public beach & G8 & 0 & 0.22 & 0.50 & 8.50 & 67.95 & 22.28 & 0.42 & 0.04 \\
\hline Karasu centrel public beach & G9 & 0 & 0.42 & 1.42 & 19.16 & 71.87 & 7.10 & 0.01 & 0 \\
\hline Kay?khane beach & G10 & 0 & 0.42 & 13.40 & 40.89 & 34.74 & 10.24 & 0.08 & 0.04 \\
\hline Karasu-Akcakoca way beach & G11 & 0 & 0.20 & 3.21 & 52.73 & 39.52 & 4.16 & 0.03 & 0 \\
\hline Kocaali, Kadikoy village coast & G12 & 0 & 0.43 & 1.37 & 27.68 & 55.81 & 14.59 & 0.02 & 0 \\
\hline Kocaali akcakoca way beach & G13 & 0 & 0.06 & 0.06 & 2.02 & 30.68 & 65.47 & 0.60 & 0.1 \\
\hline Hasancavus village coast & G14 & 0 & 0.10 & 0.02 & 2.69 & 64.52 & 32.17 & 0.41 & 0.03 \\
\hline Akcakoca near, d-100 way beach & G15 & 0 & 0.60 & 2.85 & 44.44 & 36.85 & 14.43 & 0.38 & 0.05 \\
\hline Akcakoca center beach & G16 & 0 & 0.74 & 39.60 & 51.26 & 5.84 & 1.75 & 0.13 & 0.16 \\
\hline C?arboru factory beach & G17 & 0 & 5.45 & 1.58 & 7.24 & 20.38 & 60.10 & 3.30 & 1.29 \\
\hline Akaya village beach & G18 & 0 & 1.11 & 5.30 & 19.74 & 32.58 & 38.43 & 1.86 & 0.54 \\
\hline Eregli way coast & G19 & 0 & 3.85 & 6.10 & 13.16 & 31.20 & 41.85 & 3.45 & 0.16 \\
\hline Alapli municipal beach, area traffic & G20 & 0 & 35.87 & 7.00 & 20.27 & 24.72 & 11.11 & 0.30 & 0.08 \\
\hline Mevrealti beach & G21 & 0 & 35.43 & 0.22 & 0.63 & 5.50 & 52.09 & 3.86 & 1.90 \\
\hline Erdemir public beach & $\mathrm{G} 22$ & 0 & 0.17 & 0.15 & 2.06 & 86.39 & 10.57 & 0.36 & 0.27 \\
\hline
\end{tabular}


TABLE-3

XRF RESULTS OF DUNE SEDIMENTS FROM THE SAKARYA CANYON SHORELINE (\% and ppm)

\begin{tabular}{|c|c|c|c|c|c|c|c|c|c|c|c|c|c|c|c|c|c|c|c|c|}
\hline Sample & $X$ & $\mathrm{Y}$ & $\begin{array}{l}\mathrm{Mg} \\
(\%)\end{array}$ & $\begin{array}{c}\mathrm{Al} \\
(\%)\end{array}$ & $\begin{array}{c}\mathrm{Ti} \\
(\%)\end{array}$ & $\begin{array}{l}\text { Mn } \\
(\%)\end{array}$ & $\begin{array}{c}\mathrm{Fe} \\
(\%)\end{array}$ & V & $\mathrm{Cr}$ & Co & $\mathrm{Ni}$ & $\mathrm{Cu}$ & $\mathrm{Zn}$ & $\mathrm{Ga}$ & As & $\mathrm{Zr}$ & $\mathrm{Nb}$ & $\mathrm{Cd}$ & $\mathrm{Sn}$ & $\mathrm{Pb}$ \\
\hline G1 & 71455 & 565614 & 0.71 & 5.62 & 0.18 & 0.036 & 1.53 & 27.5 & 47.9 & 8.40 & 39.3 & 5.20 & 286 & 9.00 & 10.8 & 95.6 & .40 & 2.40 & 1.40 & 25.1 \\
\hline $\mathrm{G} 2$ & 78079 & 300 & 01 & 4.78 & 0.23 & 050 & 1.58 & 34.0 & 3.6 & 9.90 & 50.7 & 6.00 & 7.9 & 7.70 & 9.30 & 66.2 & 80 & 1.90 & .80 & 11.8 \\
\hline G3 & 84186 & 4559 & 0.99 & 4.64 & 0.19 & 0.047 & 1.54 & .2 & 6.9 & 9.00 & 5.1 & 6.30 & 2.9 & & .2 & 6 & & 50 & & 11.7 \\
\hline G4 & 8 & 69 & 0.88 & 5.01 & 0.19 & 0.045 & 1.46 & 32.3 & 62. & & 49.3 & 10 & 54.7 & & 13.2 & 74.0 & 6.20 & 70 & 90 & 13.4 \\
\hline G5 & 00208 & 4556094 & 1.02 & 4.95 & 0.25 & 0.050 & 1.68 & 36.3 & 87.5 & 7.90 & 49.4 & 7.70 & 57.6 & 8.00 & 7.80 & 79.1 & 7.00 & 2.40 & 4.50 & 10.0 \\
\hline G6 & 575 & 4555 & 1.28 & 4.83 & 0.31 & 0.059 & 1.99 & & 126 & & 60.7 & & 75.4 & 60 & 80 & 74.3 & 30 & 70 & & 12.3 \\
\hline G7 & 12 & 4555929 & 1.73 & 4.79 & 0.54 & 0.084 & 3.05 & 86.2 & 469 & .6 & 78.3 & 10.2 & 133 & 10 & 9.10 & 79.8 & 13.0 & 1.60 & 5.80 & 16.0 \\
\hline G8 & 20 & 455 & 98 & 5.14 & 0.24 & 0.050 & 1.67 & 37.4 & 67.0 & 9.40 & 46.7 & 7. & 163 & & .40 & 78.7 & & 4.30 & & 16.1 \\
\hline G9 & 0309312 & 4552272 & 1.15 & 4.59 & 0.26 & 0.051 & 1.80 & 41.3 & 13.2 & 9.30 & 58.1 & 8.70 & 60.7 & 7.50 & 9.00 & 74.6 & 6.70 & 2.30 & 2.70 & 11.4 \\
\hline G10 & & 78 & 0.50 & 4.39 & 0.16 & 0.048 & 1.61 & 34.1 & 32.9 & 8. & 19. & 5.10 & 83.5 & 7. & 7.10 & 107 & .30 & 2.20 & 3.50 & 12.1 \\
\hline G11 & 15673 & 4550291 & 0.64 & 5.13 & 0.21 & 0.057 & 2.13 & 46.4 & 34.9 & 9.30 & 17.4 & 6.20 & 112 & 40 & 10.8 & 101 & 20 & 2.50 & 20 & 11.8 \\
\hline G12 & 0321943 & 4549 & 0.66 & 5.29 & 0.20 & 0.053 & 2.10 & 47.0 & 44.5 & 8.20 & 16.3 & 5.10 & 51.2 & 8.50 & 7.70 & 99.7 & 5.40 & 3.20 & 2.60 & 7.40 \\
\hline G13 & & 4549 & 4.07 & 4.74 & 1.38 & 0.182 & 7.59 & 268 & 632 & 26.0 & 48.5 & 8.50 & 71.6 & 11.3 & 6.70 & 116 & & 3.30 & 4.30 & 9.40 \\
\hline G14 & 0333211 & 4548928 & 0.91 & 4.11 & 0.26 & 0.062 & 2.35 & 53.6 & 63.4 & 8.00 & 15.8 & 5.10 & 163 & 7.00 & 8.20 & 90.3 & 5.40 & 1.40 & 3.80 & 12.6 \\
\hline G15 & 0337529 & 4549332 & 0.46 & 3.49 & 0.16 & 0.039 & 1.60 & 30 & 19.4 & 4. & 9.10 & 5. & 44.5 & 5.8 & 9.20 & 88.5 & 4.50 & 3.20 & 3.00 & 5.10 \\
\hline G16 & 0342810 & 4550607 & 0.59 & 4.29 & 0.22 & 0.057 & 2.47 & 45.3 & 28.6 & 6.80 & 9.90 & 7.80 & 44.6 & 8.20 & 7.70 & 122 & 6.60 & 2.90 & 3.50 & 6.90 \\
\hline G17 & 0348600 & 4551127 & 1.16 & 3.74 & 0.24 & 0.058 & 2.30 & 49.4 & 84.6 & 7.70 & 13.6 & 7.10 & 52.4 & 6.40 & 6.30 & 95.7 & 5.90 & 3.20 & 4.20 & 8.10 \\
\hline G18 & & & 1.57 & 4.75 & 0.27 & 0.079 & 3.08 & 67.0 & 62.6 & 9.90 & 16.9 & 13.4 & 61.3 & 9.60 & 9.90 & 116 & 6.40 & 2.30 & 2.70 & 7.50 \\
\hline G19 & 0357911 & 4553703 & 1.54 & 5.72 & 0.41 & 0.088 & 4.04 & 98.5 & 86.1 & 12.5 & 20.9 & 16.7 & 74.4 & 12.4 & 8.20 & 151 & 9.60 & 2.90 & 3.00 & 9.90 \\
\hline G20 & 0360347 & 4555238 & 0.83 & 6.21 & 0.32 & 0.077 & 3.26 & 71.0 & 39.3 & 9.70 & 18.2 & 18.2 & 74.8 & 12.8 & 12.7 & 163 & 9.10 & 2.00 & 6.20 & 11.0 \\
\hline G21 & 0365793 & 4562516 & 3.09 & 5.31 & 0.46 & 0.124 & 4.66 & 16.7 & 14.0 & 22.1 & 29. & 14.3 & 71.0 & 10.8 & 7.00 & 87.1 & 6.20 & 5.50 & 2.70 & 9.40 \\
\hline G22 & 0366628 & 4563646 & 1.20 & 7.44 & 0.29 & 0.090 & 2.88 & 79.3 & 45.7 & 15.2 & 19.6 & 15.4 & 78.3 & 12.8 & 7.80 & 10.4 & 7.20 & 5.80 & 2.50 & 37.7 \\
\hline
\end{tabular}

TABLE-4

SIMPLE STATISTICAL PARAMETERS COMPUTED FROM THE CHEMICAL DATA

\begin{tabular}{|c|c|c|c|c|c|c|c|c|c|}
\hline \multicolumn{2}{|l|}{ (2) } & $\mathrm{Mg}$ & $\mathrm{Al}$ & $\mathrm{Ti}$ & $\mathrm{Mn}$ & $\mathrm{Fe}$ & $\mathrm{V}$ & $\mathrm{Cr}$ & $\mathrm{Co}$ \\
\hline \multicolumn{2}{|l|}{ N Valid } & 22 & 22 & 22 & 22 & 22 & 22 & 22 & 22 \\
\hline \multicolumn{2}{|l|}{ Missing } & 0 & 0 & 0 & 0 & 0 & 0 & 0 & 0 \\
\hline \multicolumn{2}{|c|}{ Mean } & 1.22 & 4.95 & 0.31 & 0.06 & 2.56 & 65.04 & 110.74 & 10.76 \\
\hline \multicolumn{2}{|c|}{ Std. error of Mean } & 0.18 & 0.17 & 0.05 & 0.00 & 0.30 & 11.77 & 31.58 & 1.06 \\
\hline \multicolumn{2}{|c|}{ Median } & $1.00(a)$ & $4.81(a)$ & $0.24(a)$ & $0.05(a)$ & 2.11(a) & 46.70(a) & $63.50(a)$ & $9.30(a)$ \\
\hline \multicolumn{2}{|l|}{ Mode } & $0.46(b)$ & 3.49 (b) & $0.16(b)$ & 0.05 & $1.46(\mathrm{~b})$ & $27.50(b)$ & $19.40(\mathrm{~b})$ & $8.20(b)$ \\
\hline \multicolumn{2}{|c|}{ Std. Deviation } & 0.84 & 0.83 & 0.25 & 0.03 & 1.41 & 55.21 & 148.15 & 5.00 \\
\hline \multicolumn{2}{|l|}{ Skewness } & 2.40 & 1.09 & 3.73 & 2.36 & 2.43 & 2.84 & 2.95 & 1.97 \\
\hline \multicolumn{2}{|c|}{ Std. error of Skewness } & 0.49 & 0.49 & 0.49 & 0.49 & 0.49 & 0.49 & 0.49 & 0.49 \\
\hline \multicolumn{2}{|c|}{ Range } & 3.61 & 3.95 & 1.22 & 0.15 & 6.13 & 240.50 & 612.60 & 21.70 \\
\hline \multicolumn{2}{|l|}{ Minimum } & 0.46 & 3.49 & 0.16 & 0.04 & 1.46 & 27.50 & 19.40 & 4.30 \\
\hline \multicolumn{2}{|l|}{ Maximum } & 4.07 & 7.44 & 1.38 & 0.18 & 7.59 & 268.00 & 632.00 & 26.00 \\
\hline \multicolumn{2}{|c|}{ Sum } & 26.97 & 108.96 & 6.97 & 1.49 & 56.37 & 1431.00 & 2436.40 & 236.90 \\
\hline \multicolumn{2}{|c|}{ Percentiles 25} & 0.71 & 4.59 & 0.20 & 0.04 & 1.61 & 34.10 & 44.50 & 8.13 \\
\hline \multicolumn{2}{|c|}{50} & 1.00 & 4.81 & 0.24 & 0.05 & 2.11 & 46.70 & 63.50 & 9.30 \\
\hline $\mathrm{Ni}$ & $\mathrm{Cu}$ & $\mathrm{Zn}$ & $\mathrm{Ga}$ & As & $\mathrm{Zr}$ & $\mathrm{Nb}$ & $\mathrm{Cd}$ & $\mathrm{Sn}$ & $\mathrm{Pb}$ \\
\hline 22 & 22 & 22 & 22 & 22 & 22 & 22 & 22 & 22 & 22 \\
\hline 0 & 0 & 0 & 0 & 0 & 0 & 0 & 0 & 0 & 0 \\
\hline 33.76 & 8.82 & 118.56 & 8.81 & 8.72 & 96.82 & 7.45 & 3.00 & 3.24 & 12.57 \\
\hline 4.29 & 0.86 & 33.87 & 0.42 & 0.44 & 5.38 & 0.61 & 0.23 & 0.25 & 1.48 \\
\hline 25.15 (a) & $7.45(\mathrm{a})$ & $71.30(\mathrm{a})$ & $8.26(a)$ & $8.20(a)$ & $92.95(a)$ & $6.65(a)$ & $2.90(\mathrm{a})$ & 2.93(a) & $11.55(\mathrm{a})$ \\
\hline $9.10(b)$ & 5.10 & 163.00 & 7.50 & $7.70(b)$ & 116.00 & $5.40(b)$ & 3.20 & 2.70 & $9.40(\mathrm{~b})$ \\
\hline 20.15 & 4.06 & 158.88 & 2.00 & 2.06 & 25.24 & 2.87 & 1.12 & 1.18 & 6.94 \\
\hline 406.36 & 16.50 & 25245.22 & 4.02 & 4.27 & 637.11 & 8.28 & 1.26 & 1.41 & 48.27 \\
\hline 0.51 & 1.20 & 3.84 & 0.88 & 0.52 & 1.21 & 2.33 & 1.06 & 1.04 & 2.63 \\
\hline 0.49 & 0.49 & 0.49 & 0.49 & 0.49 & 0.49 & 0.49 & 0.49 & 0.49 & 0.49 \\
\hline 69.20 & 13.10 & 740.10 & 7.00 & 8.40 & 96.80 & 12.80 & 4.40 & 4.80 & 32.60 \\
\hline 9.10 & 5.10 & 42.90 & 5.80 & 4.80 & 66.20 & 4.50 & 1.40 & 1.40 & 5.10 \\
\hline 78.30 & 18.20 & 783.00 & 12.80 & 13.20 & 163.00 & 17.30 & 5.80 & 6.20 & 37.70 \\
\hline 742.90 & 194.10 & 2608.50 & 193.90 & 191.90 & 2130.20 & 164.00 & 66.20 & 71.30 & 276.70 \\
\hline 49.40 & 10.20 & 112.00 & 9.60 & 9.90 & 107.00 & 8.30 & 3.50 & 3.80 & 11.84 \\
\hline
\end{tabular}




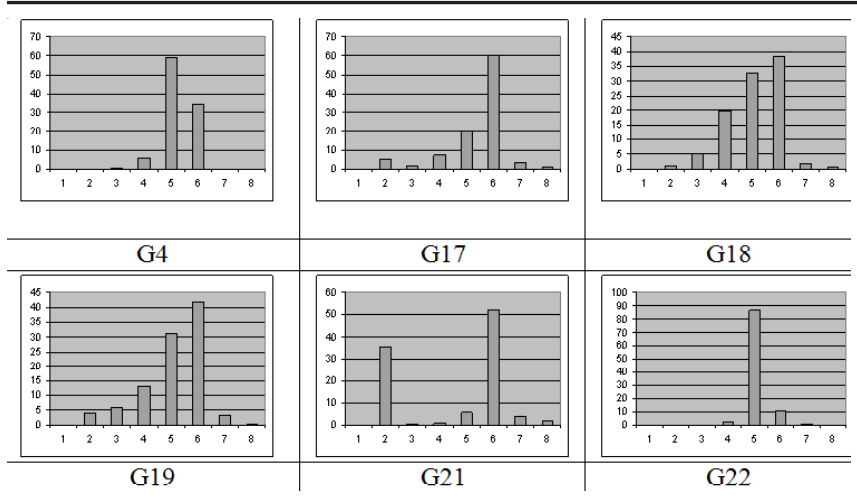

Fig. 2. Particle size distribution graphs. (1: Gravel, 2: Coarse gravel-pebble, 3: Very coarse sand, 4: coarse sand, 5: Medium sand, 6: Fine sand, 7: Very fine sand, 8: Silt + clay)

and show the toxic effect level according to different studies. Furthermore, $\mathrm{Cu}, \mathrm{Zr}$, Sn at G20; Al, Zn, Ga, Cd, Rb at G22; As at G4; maximum levels of Ni were observed at G7. Location of the $\mathrm{G} 20$ is the region near the traffic and ring road to the border and domestic waste. Erdemir location at G22, is a private beach next to the public beach limited to the highway, G4 location is near to Denizkoy (Tables 3 and 4). G7 location, Sakarya, near output of the river may be affected from the river. Study done in the Sakarya river, $\operatorname{Cr}$ (128.595 ton year ${ }^{-1}$ ) and Co (781.144 ton year $\left.{ }^{-1}\right)$ are charging the highest values. They caused heavy metal pollution. These stations showing high anomaly due to geological or anthropogenic effects.

Heavy metals showing anomaly were calculated according to the ratio of the average values of various scientific studies representing the study area (Table-5). Heavy metals in coastal sediments of Sakarya Canyon as of $\mathrm{Cr}, \mathrm{Pb}, \mathrm{Zn}, \mathrm{Cd}, \mathrm{As}, \mathrm{V}, \mathrm{Al}$, $\mathrm{Ti}$ and $\mathrm{Mn}$ have shown background values. According to earth Klark values ${ }^{27} \mathrm{Cr}, \mathrm{Pb}, \mathrm{Zn}, \mathrm{Cd}$, As; according to sandstone trace element contents ${ }^{28} \mathrm{Al}, \mathrm{Fe}, \mathrm{Mg}, \mathrm{Ti}, \mathrm{Mn}, \mathrm{Cr}, \mathrm{Ni}, \mathrm{Co}, \mathrm{Pb}, \mathrm{Zn}, \mathrm{Cd}$, As, V; according to ultrabasic rocks, trace element contents ${ }^{28}$ $\mathrm{Al}, \mathrm{Ti}, \mathrm{Pb}, \mathrm{Zn}, \mathrm{Cd}$, As, V; according to acceptable limit values for Turkey ${ }^{29} \mathrm{Cr}, \mathrm{Ni}, \mathrm{Cd}$; according to the Kizkalesi coastal sands ${ }^{30} \mathrm{Mn}, \mathrm{Pb}, \mathrm{Zn}, \mathrm{V}$ and according to the sands of the beach Susanoglu ${ }^{31} \mathrm{Al}, \mathrm{Ti}, \mathrm{Mn}, \mathrm{Pb}, \mathrm{Zn}, \mathrm{V}$ anomaly is presented. According to Klark values, $\mathrm{Cd}(20 \%)$; according to sandstone trace element contents, $\mathrm{Cd}$ (33\%); according to ultrabasic rocks, trace element contents, $\mathrm{Pb}(12.57 \%)$; according to the Turkey acceptable limit values, $\mathrm{Cd}(3 \%)$; according to the Kizkalesi coastal sands, $(5.92 \%)$ and according to the Susanoglu coastal sands, $\mathrm{Zn}(6.58 \%)$ anomaly is presented. Heavy metal concentrations such as $\mathrm{Cd}, \mathrm{Pb}$ and $\mathrm{Zn}$ in the Sakarya Canyon beach sands are greater than all other metal contents (Fig. 3). These data are compatible with principal component analysis, dendogram of elements and heavy metals showing anomaly at locations. With regard to the analysis of correlation between, $\mathrm{Zn}$ and $\mathrm{Pb}$ are determined a strong positive relation. These origins are the same.

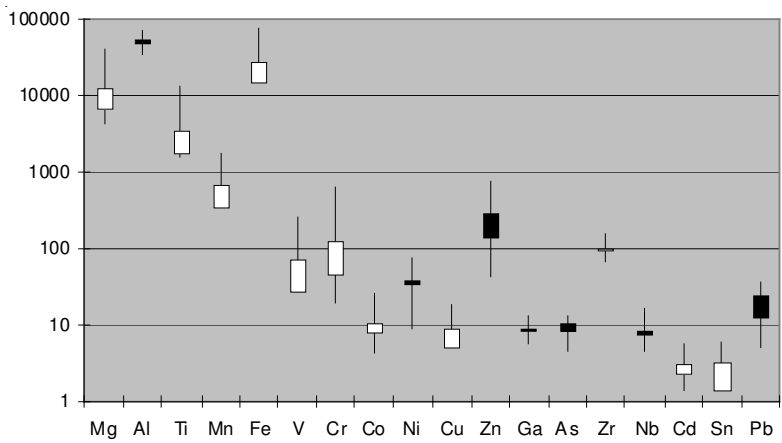

Fig. 3. Concentration levels of heavy metals

Frequency histograms of heavy metals were examined. Accordingly, Fe, Mg, Ti, Cr, Zn, Pb, Cu, Mn, Sc, Nd, Ce, Nb and $\mathrm{V}$ elements were generally concentrated in the first part of the histogram. These values have come from close distances. However, Sn, Sr, Zr, Y, Ga, Ni, Co, Cd, La, Th and P the first sections of and middle parts of the histogram; As and Al was concentrated in the middle sections. Histograms representing these data for the Fe, Sn and As were selected (Fig. 4).

TABLE-5

STATISTICAL SUMMARY IN THE SAKARYA CANYON BEACH AND ABUNDANCE OF HEAVY ELEMENTS IN EARTH CRUST, SANSTONE, THEIR ACCEPTABLE LIMITS FOR TURKEY, KIZKALESI AND SUSANOGLU BEACH SEDIMAN: BOLD CHARACTERS ARE ANOMALY VALUE, SEDIMENT (mg/kg, $\mathrm{n}=22$ ); VALID N (LISTWISE) 22; MIN MINIMUM; MAX MAXIMUM

\begin{tabular}{|c|c|c|c|c|c|c|c|c|c|c|c|c|c|}
\hline & $\begin{array}{c}\text { Sakarya } \\
\text { Canyon } \\
\text { mean (A) }\end{array}$ & $\begin{array}{c}\text { Earth } \\
\text { crust } \\
(\mathrm{mg} / \mathrm{kg})^{27} \\
\text { (B) }\end{array}$ & $\begin{array}{l}\text { Variation of } \\
\text { average } \\
\text { concentration } \\
\text { in Earth crust } \\
\text { (fold) }(\mathrm{A} / \mathrm{B})\end{array}$ & $\begin{array}{l}\text { Sandstone } \\
\text { (C) }\end{array}$ & $\begin{array}{l}\text { Variation of } \\
\text { average } \\
\text { concentration } \\
\text { in Sanstone } \\
\text { (fold) } \\
\text { (A/C) }\end{array}$ & $\begin{array}{l}\text { Ultrabasic } \\
\text { (D) }\end{array}$ & $\begin{array}{l}\text { Variation of } \\
\text { average } \\
\text { concentration } \\
\text { in Ultrabasic } \\
\text { (fold) (A/D) }\end{array}$ & $\begin{array}{l}\text { Acceptable } \\
\text { limit for } \\
\text { Turkey } \\
(\mathrm{mg} / \mathrm{kg})^{29} \\
\text { (E) }\end{array}$ & $\begin{array}{c}\text { Variation of } \\
\text { average } \\
\text { concentration } \\
\text { in TKKY } \\
\text { (fold) } \\
\text { (A/E) }\end{array}$ & $\begin{array}{l}\text { Kizkalesi } \\
\text { Beach } \\
\text { sediman } \\
\operatorname{Mean}^{30}(F)\end{array}$ & $\begin{array}{l}\text { Variation of } \\
\text { average } \\
\text { concentration } \\
\text { in Kizkalesi } \\
\text { Beach dune } \\
\text { (A/F) }\end{array}$ & $\begin{array}{l}\text { Susanoglu } \\
\text { Beach } \\
\text { sediman } \\
\text { Mean }^{31} \\
\text { (G) }\end{array}$ & $\begin{array}{c}\text { Variation of } \\
\text { average } \\
\text { concentration } \\
\text { in Susanoglu } \\
(\mathrm{A} / \mathrm{G})\end{array}$ \\
\hline $\mathrm{Al}$ & 49951 & 81000 & 0.61 & 25000 & 1.99 & 20000 & 2.49 & - & - & 8267.17 & 6.04 & 11924.24 & 4.18 \\
\hline $\mathrm{Fe}$ & 27246 & 54000 & 0.50 & 9800 & 2.78 & 94300 & 0.28 & - & - & 18803.63 & 1.44 & 13909.09 & 1.95 \\
\hline $\mathrm{Ca}$ & 43582 & 41000 & 1.06 & 39100 & 1.11 & 25000 & 1.74 & - & - & 233647.98 & 0.18 & 174745.45 & 0.24 \\
\hline $\mathrm{Mg}$ & 13121 & 23000 & 0.57 & 7000 & 1.87 & 204000 & 0.06 & - & - & 34993.03 & 0.37 & 15624.24 & 0.83 \\
\hline $\mathrm{Na}$ & 15333 & 24000 & 0.63 & 3300 & 4.65 & 4200 & 3.65 & 125 & 122.66 & 3385.59 & 4.52 & 3636.36 & 4.21 \\
\hline $\mathrm{K}$ & 11663 & 21000 & 0.55 & 10700 & 1.09 & 40 & 291.57 & - & - & 1486.73 & 7.84 & 6560.61 & 1.77 \\
\hline $\mathrm{Ti}$ & 3544 & 5 & 708.8 & 1500 & 2.36 & 300 & 11.81 & - & - & 813.48 & 4.35 & 736.36 & 4.81 \\
\hline $\mathrm{Mn}$ & 710 & 1000 & 0.71 & 90 & 7.89 & 1620 & 0.43 & - & - & 585.99 & 1.21 & 333.85 & 2.12 \\
\hline $\mathrm{Cr}$ & 110.74 & 100 & 1.10 & 35 & 3.16 & 1600 & 0.06 & 100 & 1.11 & 553.84 & 0.19 & 428.06 & 0.25 \\
\hline $\mathrm{Cu}$ & 8.82 & 50 & 0.17 & 9 & 0.98 & 10 & 0.88 & $50-140$ & 0.17 & 10.13 & 0.88 & 12.81 & 0.67 \\
\hline $\mathrm{Ni}$ & 33.76 & 75 & 0.45 & 2 & 16.88 & 2000 & 0.01 & $30-75$ & 1.12 & 186.8 & 0.18 & 145.52 & 0.23 \\
\hline Co & 10.76 & 20 & 0.54 & 0.3 & 35.87 & 150 & 0.07 & 20 & 0.53 & 28.2 & 0.38 & 21.41 & 0.51 \\
\hline $\mathrm{Pb}$ & 12.57 & 12.5 & 1.01 & 7 & 1.79 & 1 & 12.57 & $50-300$ & 0.25 & 4.55 & 2.51 & 5.51 & 2.09 \\
\hline As & 8.72 & 1.8 & 4.84 & 1 & 8.72 & 1 & 8.72 & 20 & 0.43 & 24.74 & 0.34 & 19.91 & 0.43 \\
\hline V & 65.04 & 110 & 0.59 & 20 & 3.252 & 40 & 1.626 & - & - & 63.3 & 1.03 & 38.12 & 1.71 \\
\hline
\end{tabular}




\begin{tabular}{|c|c|c|c|c|c|c|c|c|c|c|c|c|c|c|c|c|c|c|c|c|c|c|c|c|c|c|c|}
\hline & $\mathrm{Na}$ & $\mathrm{Mg}$ & $\mathrm{Al}$ & S? & $\mathrm{P}$ & $\mathrm{K}$ & $\mathrm{Ca}$ & $\mathrm{T}$ ? & $\mathrm{Mn}$ & $\mathrm{Fe}$ & Sc & V & $\mathrm{Cr}$ & Co & $\mathrm{N}$ ? & $\mathrm{Cu}$ & $\mathrm{Zn}$ & $\mathrm{Ga}$ & As & $\mathrm{Rb}$ & $\mathrm{Sr}$ & $\mathrm{Y}$ & $\mathrm{Zr}$ & $\mathrm{Nb}$ & $\mathrm{Cd}$ & $\mathrm{Sn}$ & $\mathrm{Ba}$ \\
\hline $\mathrm{Na}$ & 1 & - & - & - & - & - & - & - & - & - & - & - & - & - & - & - & $\begin{array}{lll}- & \end{array}$ & $\begin{array}{ll}- & \text { r }\end{array}$ & - & - & - & - & - & - & - & - & $\begin{array}{ll}- \\
-\end{array}$ \\
\hline $\mathrm{Mg}$ & $-.524\left(^{*}\right)$ & 1 & - & - & - & - & - & - & - & - & - & - & - & - & - & - & . & 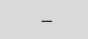 & . & - & - & - & - & - & - & - & - \\
\hline $\mathrm{Al}$ & $.602(* *)$ & ) .089 & 1 & - & - & - & - & - & - & - & - & - & - & - & - & - & - & - & - & - & - & - & - & - & - & - & - \\
\hline S? & .127 & $.742(* *)$ & )$^{-.474(*)}$ & 1 & - & $\begin{array}{lll}- & \text { r }\end{array}$ & - & $\begin{array}{lll}- & \end{array}$ & - & - & - & - & - & - & - & - & - & $\begin{array}{lll}- & \\
\end{array}$ & $\begin{array}{lll}- & \\
\end{array}$ & - & - & - & - & - & - & - & - \\
\hline $\mathrm{P}$ & .086 & .057 & $.623(* *)$ & ) -295 & 1 & - & - & - & - & - & - & - & - & - & - & - & - & - & - & - & - & - & - & - & - & - & - \\
\hline $\mathrm{K}$ & $.539(* *)$ & $-439(*)$ & $.556(* *)$ & ) -196 & .364 & 1 & - & - & - & - & - & - & - & - & - & - & - & - & - & - & - & - & - & - & - & - & - \\
\hline $\mathrm{Ca}$ & -.256 & $.700(*)$ & .113 & $.904(* *)$ & -.047 & .062 & 1 & - & - & - & - & - & - & - & - & - & - & - & - & - & - & - & - & - & - & - & - \\
\hline $\mathrm{T} ?$ & $.589^{(* *)}$ & $.892(* *)$ & .052 & $.671^{-* *}$ & .067 & $-.456\left(^{*}\right)$ & $.619(* *)$ & 1 & - & - & - & - & - & - & - & - & - & - & - & - & - & - & - & - & - & - & - \\
\hline $\mathrm{Mn}$ & $-.447(*)$ & $.934(* *)$ & .234 & $.648(* *)$ & .214 & $\left.-.4911^{*}\right)$ & $\left..5022^{*}\right)$ & $.914(* *)$ & 1 & - & - & - & - & - & - & - & - & - & - & - & - & - & - & - & - & - & - \\
\hline $\mathrm{Fe}$ & $-.525(*)$ & $.901(* *)$ & .181 & $.577(* *)$ & .225 & $-.503(*)$ & .417 & $.916(* *)$ & $.981(* *)$ & 1 & - & - & - & - & - & - & - & - & - & - & - & - & - & - & - & - & - \\
\hline Sc & $.573(* *)$ & $.966(* *)$ & .100 & $.7311^{(* *)}$ & .084 & $-.427\left(^{*}\right)$ & $.668(* *)$ & $.956(* *)$ & $.948(* *)$ & $.937(* *)$ & 1 & - & - & - & - & - & - & - & - & - & - & - & - & - & - & - & - \\
\hline $\mathrm{v}$ & $-.490\left(^{*}\right)$ & $.949(* *)$ & .159 & $.6477^{(* *)}$ & .099 & $-.509(*)$ & $.538(* *)$ & $.935(* *)$ & $.987(* *)$ & $.980(* *)$ & $.970(* *)$ & 1 & - & - & - & - & - & - & - & - & - & - & - & - & - & - & - \\
\hline $\mathrm{Cr}$ & $.5600^{(* *)}$ & $.791(* *)$ & -.074 & $.664^{(* *)}$ & -.004 & -.398 & $.697(* *)$ & $.907(* *)$ & $.751(* *)$ & $.731(* *)$ & $.811(* *)$ & $.7733^{(* *)}$ & 1 & - & - & - & - & - & - & - & - & - & - & - & - & - & - \\
\hline Сo & -.269 & $.942(* *)$ & .331 & $.802(* *)$ & .131 & -.317 & $.686(*))$ & $.852(* *)$ & $.935(* *)$ & $.875(* *)$ & $.910(* *)$ & $.931(* *)$ & $.763(* *)$ & 1 & - & - & - & - & - & - & - & - & - & - & - & - & - \\
\hline N? & -.116 & .279 & .008 & $.6477^{(* *)}$ & -.053 & .331 & $.788(* *)$ & .269 & .058 & -022 & .232 & .078 & $.525\left(^{*}\right)$ & .290 & 1 & - & - & - & - & - & - & - & - & - & - & - & - \\
\hline $\mathrm{Cu}$ & -.005 & .348 & $.629(* *)$ & ) -388 & $.775(* *)$ & .122 & .046 & .224 & $.485\left(^{*}\right)$ & $.485\left(^{*}\right)$ & .326 & .393 & .074 & $.424\left(^{*}\right)$ & ) -.150 & 1 & - & - & - & - & - & - & - & - & - & - & - \\
\hline $\mathrm{Zn}$ & $.6499^{(* *)}$ & ) -.040 & $.698(* *)$ & -.229 & .323 & .270 & .046 & -.038 & .098 & .008 & -.060 & .020 & -.081 & .182 & -.103 & .282 & 1 & - & - & - & - & - & - & - & - & - & - \\
\hline $\mathrm{Ga}$ & .109 & $.466\left(^{*}\right)$ & $.816(* *)$ & $.567(* *)$ & $.707(* *)$ & ) .173 & .175 & $\left..4511^{*}\right)$ & $.645(* *)$ & $.645(* *)$ & $.502\left(^{*}\right)$ & $.578(* *)$ & .250 & $.6177^{(* *)}$ & ) -0.08 & $.854^{(* *)}$ & ). $\left.4377^{*}\right)$ & 1 & - & - & - & - & - & - & - & - & - \\
\hline As & .160 & -.326 & .194 & .047 & .289 & $.457(*)$ & -.142 & -.272 & -.290 & -.259 & -.280 & -.293 & -.238 & -.285 & .027 & .095 & -.054 & .080 & 1 & - & - & - & - & - & - & - & - \\
\hline $\mathrm{Rb}$ & .400 & -.417 & $.527(*)$ & -153 & $\left..4522^{*}\right)$ & .969 (**) & -.024 & -.409 & $\left.-.4411^{*}\right)$ & -.420 & -.382 & $-.462(*)$ & -388 & -.326 & .260 & .217 & .130 & .242 & $.502(*)$ & 1 & - & - & - & - & - & - & - \\
\hline $\mathrm{Sr}$ & $.536(*)$ & .168 & $.580(* *)$ & $.664^{-(* *)}$ & .080 & $.508\left(^{(*)}\right.$ & $.641(* *)$ & .028 & .059 & -.072 & .091 & .046 & .122 & .346 & $.552(* *)$ & .125 & $.620^{(* *)}$ & ) .254 & .010 & .322 & 1 & - & - & - & - & - & - \\
\hline $\mathrm{Y}$ & $-.507\left(^{*}\right)$ & $.565(* *)$ & ) .158 & -.258 & $.492(*)$ & $-435\left(^{*}\right)$ & .012 & $.674(* *)$ & $.759(* *)$ & $.838(* *)$ & $.648(* *)$ & $.726(* *)$ & $.492\left(^{*}\right)$ & $.541^{(* *)}$ & ) -287 & $.616^{(* *)}$ & ) -085 & $.670(* *)$ & -0.087 & -.279 & -.384 & 1 & - & - & - & - & - \\
\hline $\mathrm{Zr}$ & -.153 & .082 & .345 & .092 & $.566(* *)$ & -.077 & $-.434\left(^{*}\right)$ & .220 & .351 & $.466\left(^{*}\right)$ & .198 & .308 & -.023 & .117 & $.588^{(* *)}$ & $.634(* *)$ & .046 & $.670^{(* *)}$ & .103 & .103 & $-.462(*)$ & $.797(* *)$ & 1 & - & - & - & - \\
\hline $\mathrm{Nb}$ & $-.479\left(^{*}\right)$ & $.710(* *)$ & ) .193 & $.7433^{(* *)}$ & .279 & -.112 & $.642(* *)$ & $.8866^{(* *)}$ & $.734(* *)$ & $.748(* *)$ & $.788(* *)$ & $.739(* *)$ & $.897(* *)$ & ). $720^{(* *)}$ & ). $470(*)$ & ) .283 & .036 & $.510(*)$ & -.132 & -.064 & .163 & $.618(* *)$ & .253 & 1 & - & - & - \\
\hline $\mathrm{Cd}$ & .339 & .305 & .418 & -.298 & -.065 & -.006 & .233 & .086 & .286 & .199 & .213 & .275 & -.061 & .392 & -.069 & .271 & $\left..4599^{*}\right)$ & .301 & -.282 & -.114 & $.501\left(^{*}\right)$ & -.091 & -.123 & -.073 & 1 & - & - \\
\hline $\mathrm{Sn}$ & -.421 & .165 & -.092 & -.046 & .255 & -.292 & -.026 & .337 & .307 & .333 & .239 & .267 & .409 & .162 & .005 & .303 & -.201 & .215 & .048 & -.197 & -.303 & $.560(* *)$ & .393 & .401 & $-.452\left(^{*}\right)$ & 1 & - \\
\hline $\mathrm{Ba}$ & $\left..519{ }^{*}\right)$ & -296 & $.663(* *)$ & ) -.284 & $.508\left(^{*}\right)$ & $\left..9566^{* * *}\right)$ & .080 & -.380 & -.332 & -.342 & -.310 & -.368 & -.375 & -.172 & .243 & .326 & .351 & .359 & $.425\left(^{*}\right)$ & $.941(* *)$ & $.526(*)$ & -.276 & .062 & -.058 & .069 & -.309 & 1 \\
\hline $\mathrm{La}$ & .002 & .401 & $.510(*)$ & $.669(* *)$ & .365 & .192 & $\left..5122^{*}\right)$ & $.523(*)$ & $.479\left(^{*}\right)$ & $.428\left(^{*}\right)$ & $.489\left(^{*}\right)$ & $\left..4566^{*}\right)$ & $.509(*)$ & $.5388^{(* *)}$ & ) .356 & .307 & .345 & $.546(* *)$ & .323 & .162 & $.461\left(^{*}\right)$ & .313 & .142 & $.633(* *)$ & ) .080 & .321 & .219 \\
\hline $\mathrm{Ce}$ & -.328 & $.669(* *)$ & .278 & $.7300^{(* *)}$ & .226 & -.107 & $.626(* *)$ & $.858(* *)$ & $.723(* *)$ & $.724(* *)$ & $.777(* *)$ & $.740(* *)$ & $.801(* *)$ & ). $720(* *)$ & ) .349 & .209 & .223 & $.519\left(^{*}\right)$ & -.050 & -.100 & .256 & $\left..5344^{*}\right)$ & .214 & $.902(* *)$ & ) .075 & .210 & -.062 \\
\hline $\mathrm{Nd}$ & $-.456\left(^{*}\right)$ & . $698(* *)$ & .236 & $.5711^{(* *)}$ & .353 & -.301 & .397 & $.884(* *)$ & $.824(* *)$ & $.858(* *)$ & $.797(* *)$ & .820 (**) & $.797(* *)$ & $.712(* *)$ & ) .106 & .376 & .083 & $.620(* *)$ & -.050 & -.227 & -.033 & $.808(* *)$ & $\left..4833^{*}\right)$ & $.885(* *)$ & ) - -011 & $.452\left(^{*}\right)$ & ) -217 \\
\hline $\mathrm{Pb}$ & $.674(* *)$ & ) -.074 & $.715(* *)$ & ) -349 & .293 & $.499\left(^{*}\right)$ & .194 & -.070 & -.004 & - 100 & -.096 & -.064 & -.042 & .165 & .152 & .188 & $.9377^{(* *)}$ & ) .383 & .074 & 349 & $.7577^{(* *)}$ & -233 & -.088 & .104 & .367 & -.254 & .547 (* $^{\circ}$ \\
\hline Th & -.094 & .182 & .376 & -.391 & $.436\left(^{*}\right)$ & .283 & .173 & .398 & .288 & .363 & .295 & .302 & .395 & .261 & .121 & .277 & .104 & $.529\left(^{*}\right)$ & .267 & .353 & .072 & $\left..4922^{*}\right)$ & $.514\left(^{*}\right)$ & $.6599^{* * *}$ & ) -.132 & .226 & .321 \\
\hline
\end{tabular}




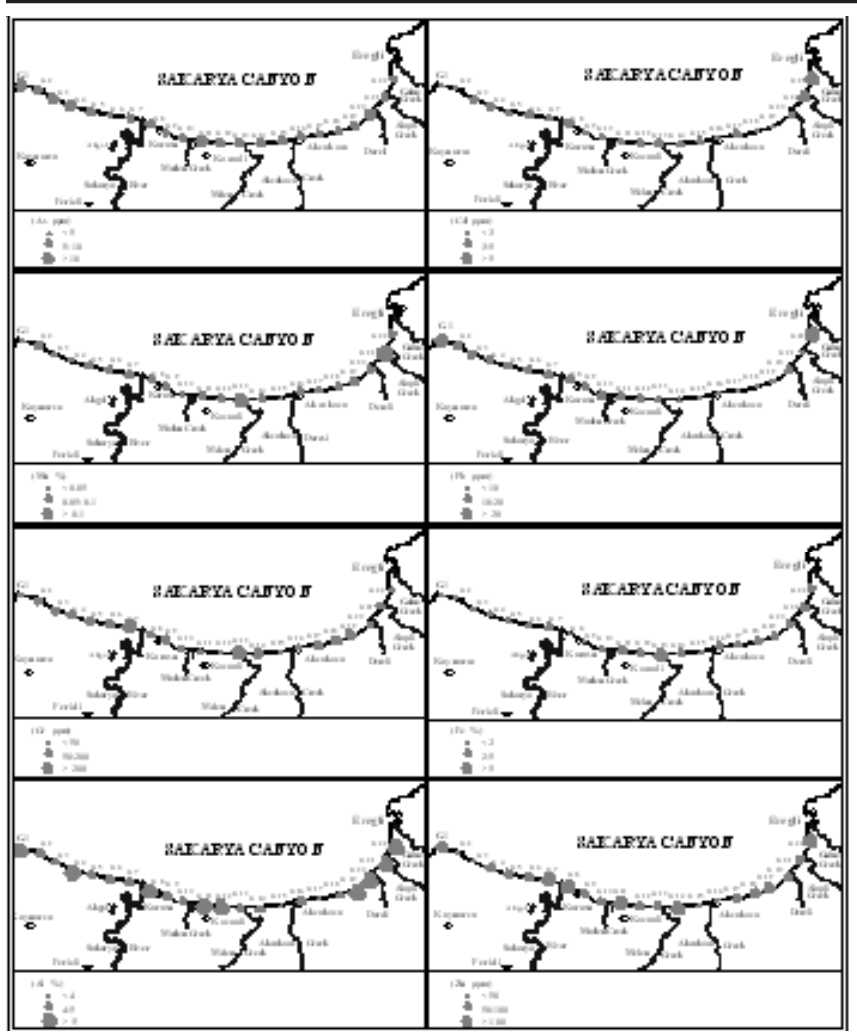

Fig. 4. Distribution map of some heavy metal contents map beach sand samples at the Sakarya Canyon

Heavy metals offering toxic features (Mg, Ti, Mn, Fe, V, $\mathrm{Cr}, \mathrm{Co}, \mathrm{Nb}, \mathrm{Al}, \mathrm{Cu}, \mathrm{Zn}, \mathrm{Ga}, \mathrm{Pb}, \mathrm{Ti}$ and $\mathrm{Zr}$ ) are examined to the correlation relationship. With reference to the correlation matrix (Table-6) which indicates the correlation among different elements, the positive high relationship $\left(r^{2}=\right.$ less than 0.10$)$ among sand vs. contaminated elements (between $\mathrm{Mg}$ and $\mathrm{Ti}$, $\mathrm{Mn}, \mathrm{Fe}, \mathrm{V}, \mathrm{Cr}, \mathrm{Co}, \mathrm{Nb}$; between $\mathrm{Al}$ and $\mathrm{Cu}, \mathrm{Zn}, \mathrm{Ga}, \mathrm{Pb}$; between $\mathrm{Ti}$ and $\mathrm{Mn}, \mathrm{Fe}, \mathrm{V}, \mathrm{Cr}, \mathrm{Co}, \mathrm{Nb}$; between $\mathrm{Mn}$ and $\mathrm{Fe}, \mathrm{V}, \mathrm{Cr}, \mathrm{Co}$, $\mathrm{Ga}, \mathrm{Nb}$; between $\mathrm{Fe}$ and $\mathrm{V}, \mathrm{Cr}, \mathrm{Co}, \mathrm{Ga}, \mathrm{Nb}$; between $\mathrm{V}$ and $\mathrm{Cr}$, $\mathrm{Co}, \mathrm{Ga}, \mathrm{Nb}$; between $\mathrm{Cr}$ and $\mathrm{Co}$; between $\mathrm{Ni}$ and $\mathrm{Zr}$; between $\mathrm{Cu}$ and $\mathrm{Ga}, \mathrm{Zr}$; between $\mathrm{Zn}$ and $\mathrm{Pb}$; between $\mathrm{Ga}$ and $\mathrm{Zr}$ ) indicates the presence of these elements in sand layers. The negative high relationship $\left(\mathrm{r}^{2}=\right.$ less than 0.10$)$ among sand $v s$. contaminated elements (between $\mathrm{Co}$ and $\mathrm{Zr}$ ) indicate the presence of these elements in sand layers. They come from different sources or industrial input/activities of pollution in the study area.

With regard to principal component analysis, three factors are determined as F1, F2, F3. The first factor (Factor 1) explains $40.911 \%$ of the total variance with a high eigenvalue of 13.092 (Table-7). The first factor can be termed as "natural process factor". The second factor (Factor 2) explains 21.558 $\%$ of the total variance with an eigen value of 6.899 (Table-7). This factor can be termed as "anthropogenic factor". The third factor (Factor 3) explains $13.548 \%$ of total variance with an eigenvalue of 4.335 (Table-7). This factor can be termed as "intermediate factor". All elements in the Sakarya Canyon are represented by the three component factors. According to PCA analysis using the three factors, the F1 factor contains $\mathrm{Mg}$, Ti, $\mathrm{Mn}, \mathrm{Fe}, \mathrm{V}, \mathrm{Cr}$, Co and $\mathrm{Nb}$. F2 factor contains $\mathrm{Al}, \mathrm{Zn}$ and $\mathrm{Pb}$. F3 factor contains $\mathrm{Zr}$ (Table-8).

\begin{tabular}{cccc}
\multicolumn{3}{c}{ TABLE-7 } \\
\multicolumn{3}{c}{ EXPLANATION OF TOTAL VARIANCE OF } \\
SEDIMENTS WITH EIGEN VALUES (PCA) \\
\hline \multirow{2}{*}{ Component } & \multicolumn{3}{c}{ Initial eigen values } \\
\cline { 2 - 4 } & Total & Variance (\%) & Cumulative (\%) \\
\hline 1 & 13.092 & 40.911 & 40.911 \\
2 & 6.899 & 21.558 & 62.469 \\
3 & 4.335 & 13.548 & 76.018 \\
\hline Extraction method: Principal component analysis (PCA).
\end{tabular}

TABLE-8

RESULTS OF FACTOR ANALYSIS FOR BEACH SEDIMENTS IN THE SAKARYA CANYON

\begin{tabular}{cccc} 
& \multicolumn{3}{c}{ Rotated component matrix (a) } \\
\cline { 2 - 4 } $\mathrm{Mg}$ & 1 & 2 & 3 \\
$\mathrm{Al}$ & 0.903 & -0.164 & -0.229 \\
$\mathrm{Ti}$ & 0.272 & 0.870 & 0.210 \\
$\mathrm{Mn}$ & 0.953 & -0.210 & -0.112 \\
$\mathrm{Fe}$ & 0.953 & -0.123 & 0.039 \\
$\mathrm{~V}$ & 0.944 & -0.183 & 0.156 \\
$\mathrm{Cr}$ & 0.948 & -0.181 & -0.022 \\
$\mathrm{Co}$ & 0.852 & -0.212 & -0.291 \\
$\mathrm{Ni}$ & 0.915 & 0.055 & -0.217 \\
$\mathrm{Cu}$ & 0.246 & 0.239 & -0.690 \\
$\mathrm{Zn}$ & 0.467 & 0.411 & 0.533 \\
$\mathrm{Ga}$ & 0.097 & 0.671 & -0.044 \\
$\mathrm{As}$ & 0.661 & 0.534 & 0.444 \\
$\mathrm{Zr}$ & -0.194 & 0.363 & 0.270 \\
$\mathrm{Nb}$ & 0.332 & 0.056 & 0.909 \\
$\mathrm{Cd}$ & 0.892 & 0.067 & -0.061 \\
$\mathrm{Sn}$ & 0.176 & 0.297 & -0.284 \\
$\mathrm{~Pb}$ & 0.369 & -0.253 & 0.405 \\
& 0.066 & 0.806 & -0.193 \\
\hline
\end{tabular}

Extraction method: Principal component analysis. Varimax with Kaiser Normalization. (a): 3 components extracted.

The Hierarchical cluster analysis dendogram was conducted in order to determine similarities between the stations. Hierarchical group which is done, $50 \%$ of Q-type cluster is a similarity level. It was seen that the number of stations were sufficient for analysis. According to dendogram, there are similarities among G-3 4, 2, 5, 6, 9, 8 and between G19 and 20; among G10, 11, 12, 16, 17, 15, 18, 14; between G7 and 13. Although G21, G22 and G1 are the stand-alone stations, they are not similar to the other stations. The groups in the stations represent pollution. However, the stations that are not similar to each other have a unique situation (Fig. 5). As listed the first to these $\mathrm{Mn}, \mathrm{V}, \mathrm{Fe}, \mathrm{Mg}, \mathrm{Co}, \mathrm{Cr}, \mathrm{Nb}$; the second $\mathrm{Pb}$, $\mathrm{Zn}, \mathrm{Al}$; the third dendogram connected $\mathrm{Cd}$ from the outside (Fig. 6). Dendogram of an element consistent with factor analysis.

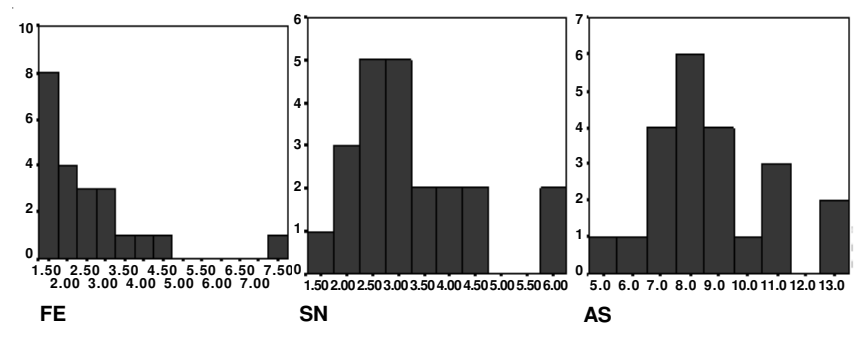

Fig. 5. Frequency histograms of heavy metal contents in the study area (Fe, Sn, As) 
RestanceDistanceClusterCombine

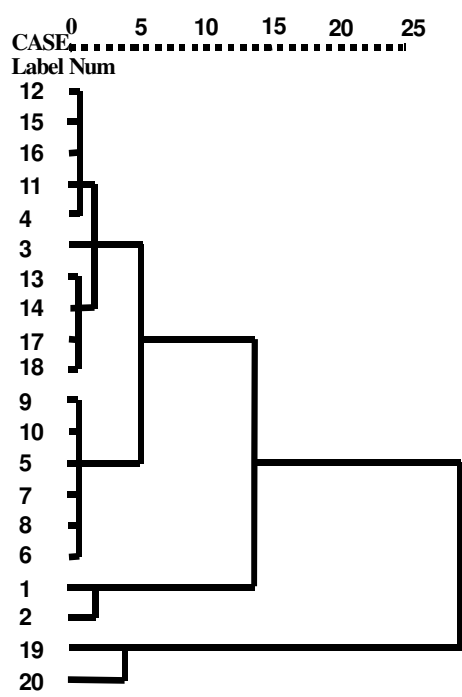

Fig. 6. Hierarchical cluster analysis dendogram

The first of these can be listed as: $\mathrm{Mn}, \mathrm{V}, \mathrm{Fe}, \mathrm{Mg}, \mathrm{Co}, \mathrm{Cr}$, $\mathrm{Nb}$; the second; $\mathrm{Pb}, \mathrm{Zn}, \mathrm{Al}$; and the third can be listed as the dendogram connected to Cd externally (Fig. 7). Element Dendogram is consistent with factor analysis. XRF results were calculated according to the Model Summary and Anova (Table-9), in the calculation, the data were examined according to $\mathrm{Fe}$ at calculation. Percentage of explanatory regression equation is $\mathrm{R}^{2}=100 \%$ so there is no error margin. The results of the analysis are highly accurate. Explanatory variables explain the exchange of Fe element 21 (Cr, P, Cd, As, Pb, Ni, Th, Sn, La, $\mathrm{Na}, \mathrm{Ga}, \mathrm{Ca}, \mathrm{Ce}, \mathrm{Cu}, \mathrm{Rb}, \mathrm{Co}, \mathrm{Zr}, \mathrm{Nd}, \mathrm{Y}, \mathrm{Mg}, \mathrm{Sr}$ ) this value is very high.

\begin{tabular}{|c|c|c|c|c|c|c|}
\hline \multicolumn{7}{|c|}{$\begin{array}{l}\text { TABLE-9 } \\
\text { MODEL SUMMARY AND ANOVA TABLES OF } \\
\text { REGRESSION DATA FROM DUNE SEDIMENTS }\end{array}$} \\
\hline \multicolumn{7}{|c|}{ Model summary } \\
\hline Model & $\mathrm{r}$ & & R Square & $\begin{array}{l}\text { Adjusted } \\
\text { R square }\end{array}$ & & $\begin{array}{l}\text { Std. error } \\
\text { of the } \\
\text { estimate }\end{array}$ \\
\hline 1 & 1.00 & (a) & 1.000 & 1.000 & & - \\
\hline \multicolumn{7}{|c|}{ ANOVA (b) } \\
\hline Model & & $\begin{array}{l}\text { Sum of } \\
\text { squares }\end{array}$ & df & $\begin{array}{l}\text { Mean } \\
\text { square }\end{array}$ & $\mathrm{F}$ & Sig. \\
\hline \multirow{3}{*}{1} & Regression & 42.085 & 21 & \multirow{3}{*}{2.004} & & \multirow{3}{*}{ (a) } \\
\hline & Residual & 0.000 & 0 & & - & \\
\hline & Total & 42.085 & 21 & & & \\
\hline
\end{tabular}

a: Predictors: (Constant), Cr, P, Cd, As, Pb, Ni, Th, Sn, La, Na, Ga, $\mathrm{Ca}, \mathrm{Ce}, \mathrm{Cu}, \mathrm{Rb}, \mathrm{Co}, \mathrm{Zr}, \mathrm{Nd}, \mathrm{Y}, \mathrm{Mg}$, Sr. b: Dependent Variable: Fe

Mnv Fe Mg Sc CoTiCrNbCeNd La YZr ThSn Ca Ni Sr KRb BaZnPb Al CuGaP Na Cd Si As

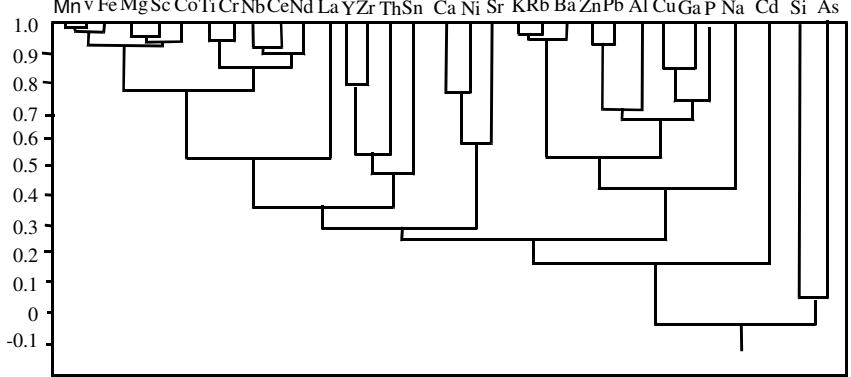

Fig. 7. Dendogram of element
Heavy metal offering anomaly should be thought to be the anthropogenic and geological origin at the Sakarya Canyon. Heavy metals such as $\mathrm{Cr}, \mathrm{Ni}$, Co may be associated with ultra-basic rocks. Anthropogenic origin anomalies as the source ports are a waste of ships, flowing rivers, industrial waste, wastewater and domestic waste and thermal power plant waste. Taking emergency measures, there is required to stop metal pollution and to form protection areas for the protection of ecological balance.

\section{ACKNOWLEDGEMENTS}

The financial support of the Scientific Research Projects Unit of Akdeniz University is gratefully acknowledged.

\section{REFERENCES}

1. O.E. Frihy and K.M. Dewidar, Marine Geol., 199, 27 (2003).

4. V.C. Lakhan, K. Cabana and P.D. LaValle, Environ. Geol., 42, 73 (2002).

5. T. Liaghati, M. Preda and M. Cox, Environ. Int., 29, 935 (2003).

2. L. Boruvka, O. Vacek and J. Jehlicka, Geoderma, 128, 289 (2005).

3. S. Topcuoglu, K.C. Guven, N. Balkis and C. Kirbasoglu, Chemosphere, 52, 1683 (2003).

6. M. Tuzen, B. Verep, A.O. Ogretmen and M. Soylak, Environ. Monit. Assessm., 151, 363 (2009).

7. O. Yigiterhan and J.W. Murray, Marine Chem., 111, 63 (2008).

8. B. Koz, N. Celik and U. Cevik, Ecol. Indicators, 10, 762 (2010).

9. H.A. Ergul, S. Topcuoglu, E.O. lmez and C. Kirbasoglu, Coastal Shelf Sci., 78, 396 (2008)

10. N. Kiratli and M. Ergin, Appl. Geochem., 2, 775 (1996).

11. S. Topcuoglu, C. Kirbasoglu and N. Gungor, Environ. Int., 27, 521 (2002).

12. F. Yucesoy and M. Ergin, Chem. Geol., 99, 265 (1992).

13. M. Duman, S. Duman, T.W. Lyons, M. AvcV, M. Izdar and E. Demirkurt, Marine Geol., 227, 51 (2006).

14. S. Isik, E. Dogan, L. Kalin, M. Sasal and N. Agiralioglu, Catena, 75, 172 (2008).

15. M. Yucel, S.K. Konovalov, T.S. Moore, C.P. Janzen and G.W. Luther, Chem. Geol., 269, 364 (2010).

16. cedgm.gov.tr; Cevresel Etki Degerlendirmesi ve Planlama Genel Mudurlugu, Sogutozu, Ankara (in Turkish).

17. E. Yigitbas, R. Kerrich, Y. Yilmaz, A. Elmas and Q.L. Xie, Precambrian Res., 132, 179 (2004).

18. P.A. Ustaomer, R. Mundil and P.R. Renne, Terra Nova, 17, 215 (2005).

19. A.M.C. Sengor and Y. Yilmaz, Tectonophysics, 75, 181 (1981).

20. A.I. Okay, E. Bozkurt, M. Satir, E. Yigitbas, Q.G. Crowley and C.K. Shang, Tectonophysics, 461, 252 (2008).

21. A.I. Okay, Anschnitt, 21, 19 (2008).

22. Y. Yilmaz, O. Tuysuz, A.M. Gozubol and E. Yigitbas, Bull. Istanbul Earth Sci. Rev., 2, 239 (1981).

23. E. Bingol, B. Akyurek, B. Korkmazer and I. Ketin, 50th Anniversary of Turkish Republic, Mineral Research and Exploration Institute Press, Ankara, p. 70 (1973).

24. O. Tekeli, Geology, 9, 68 (1981).

25. M.G. Yalcin, A. Tumuklu, M. Sonmez and D.S. Erdag, Environ. Monit. Assessm., 164, 311 (2010).

26. M.G. Yalcin, I. Narin and M. Soylak, Environ. Monit. Assessm., 128, 351 (2007).

27. K. Krauskopf, Introduction to Geochemistry, McGraw-Hill Book Company, New York, p. 123 (1979).

28. K.K. Turekian and K.H. Wedepohl, Geol. Soc. Am. Bull., 72, 175 (1961).

29. TKKY, Toprak Kirliliginin Kontrolu Yonetmeligi, 2005 tarih ve 25831 sayili Resmi Gazete, 31 Mayis, Ankara (2005) (in Turkish).

30. M.G. Yalcin and S. Ilhan, Bull. Environ. Contam. Toxicol., 81, 57 (2008).

31. M.G. Yalcin, Environ. Geol., 58, 119 (2009). 